\title{
NEUROIMAGING AND PATTERN RECOGNITION TECHNIQUES FOR AUTOMATIC DETECTION OF ALZHEIMER'S DISEASE: A REVIEW
}

\author{
Rupali Kamathe ${ }^{\mathbf{1}}$ and Kalyani Joshi ${ }^{2}$ \\ ${ }^{1}$ Department of Electronics and Telecommunication Engineering, College of Engineering, Pune, India \\ ${ }^{2}$ Department of Electronics and Telecommunication Engineering, P.E.S's Modern College of Engineering, India
}

\begin{abstract}
Alzheimer's disease (AD) is the most common form of dementia with currently unavailable firm treatments that can stop or reverse the disease progression. A combination of brain imaging and clinical tests for checking the signs of memory impairment is used to identify patients with $A D$. In recent years, Neuroimaging techniques combined with machine learning algorithms have received lot of attention in this field. There is a need for development of automated techniques to detect the disease well before patient suffers from irreversible loss. This paper is about the review of such semi or fully automatic techniques with detail comparison of methods implemented, class labels considered, data base used and the results obtained for related study. This review provides detailed comparison of different Neuroimaging techniques and reveals potential application of machine learning algorithms in medical image analysis; particularly in $A D$ enabling even the early detection of the disease- the class labelled as Multiple Cognitive Impairment.
\end{abstract}

Keywords:

Image Classification, Feature Extraction, Computer Aided Diagnosis, Image Databases, Image Analysis, Alzheimer's disease

\section{INTRODUCTION}

Alzheimer's disease (AD) is a neurodegeneration disorder that progressively declines individual's memory and cognitive skills. It is the most common type of dementia in the elderly. German Neuropathologist and psychiatrist, Alois Alzheimer was the first to describe this incurable and degenerative terminal disease in 1906 and the disease was named after him. The risk of developing $\mathrm{AD}$ increases with age. It is most often diagnosed in people over age of 65 years. However, many individuals younger than age 65 can also develop the disease. It is irreversible, and progressively destroys memory and thinking skills which results in decline of memory and mental function. Symptoms include confusion, irritability, language breakdown, aggression, mood swings, longterm memory loss and the decline of different senses of the diseased. Ultimately the result is the loss of bodily functions leading to death [15]. There are four different stages of disease progression. The first stage with a variety of symptoms (most commonly amnesia) which do not significantly alter daily life is known as Mild Cognitive Impairment (MCI). The next stages of $\mathrm{AD}$ (Mild and Moderate AD) are characterized by increasing cognitive deficits, and decreasing independence of patients (Severe AD) [33]. Between 6-25\% of people affected with MCI progress to $\mathrm{AD}$ every year.

Different neuroimaging examinations are an essential part of the diagnostic investigation of $\mathrm{AD}$. These examinations also help to search for biological markers that provide supportive diagnostic features for the AD. There is an immense need to devise automated approaches for early diagnosis and detection of $\mathrm{AD}$ as it may aid experts to prescribe medications that can at least slow down the disease progression and to help the patient and the patient's family to develop coping techniques. For this automated diagnosis, Machine learning and Computer-Aided Diagnosis (CAD) have gained increasing attention in the medical field. A machine learning algorithm is trained using a set of examples to produce a desired output. Those examples are divided into different classes. When a new instance is presented to the learning algorithm, it assigns it a class according to the set of classification rules. The only information given to the algorithm is a set of labeled examples (i.e. a set of instances with their class) [15]. Important steps in CAD include Image pre-processing followed by feature extraction such that within class similarity is maximized and between classes similarity is minimized. After feature extraction, some of the selected features from the dataset are used in the training process of the learning algorithm. In this process the aim is to find the optimal subset that increases the efficiency of the learning algorithm. The curse of dimensionality demands the dimensionality of the pattern representation should be as small as possible. Therefore, feature extraction and selection received great attention; specifically to optimize the feature set and to increase the prediction accuracy of the classification of AD stages is of great interest.

The rest of the paper is organized as follows: A current status and effects of $\mathrm{AD}$ is presented in section 2, A comprehensive literature survey of work done towards computer-aided diagnosis of $\mathrm{AD}$ is presented in section 3, section 4 provides comparison of different Neuroimaging techniques, section 5 is about Pattern Recognition techniques and section 6 is about Discussion followed by conclusions in section 7 .

\section{ALZHEIMER'S DISEASE (AD)}

$\mathrm{AD}$ is a physical brain disease that causes dementia by a progressive degeneration of brain cells. Dementia is a general term for a group of brain disorders, of which $\mathrm{AD}$ is the most common. AD accounts for 50 to 70 percent of all dementia cases. In $\mathrm{AD}$, it becomes difficult for a person to carry out daily activities. From person to person, the rate of progression greatly varies. The complete dependence of a patient is seen with disease progression. The disease is characterized by specific changes in the brain as the "plaques" are formed outside the brain cells due to an abnormal buildup of a beta amyloid protein. Also, "tangles" builds up inside the brain cells due to another protein called tau. These protein accumulations damage the connections between brain cells and thus disrupt the messages within the brain. This causes the brain cells to eventually die, thus the volume of a brain shrinks (atrophy). The brain function is affected eventually [34]. 
Mild Cognitive Impairment (MCI) is believed to be a transitional stage between normal aging and AD. During MCI a memory loss is seen in person that cannot be linked to age problems but also not severe enough to point to probable AD.

\subsection{SYMPTOMS AND HEALTH ISSUES}

The disease symptoms and its effects on each person may vary. Common symptoms may include [34]:

- Persistent and frequent memory loss

- Vagueness in conversation and language difficulties

- Difficulties in planning, organizing, problem solving capabilities and logical thinking

- Loss of enthusiasm, deterioration of social skills and unpredictable emotional/ behavioral and overall personality changes

- Becoming disoriented and longer times to do routine tasks

- Inability to process questions/ instructions etc.

\subsection{WORLDWIDE POPULATION PROGRESSION OF AD}

AND

According to the Alzheimer's Association, in 2010 there were 35.6 million people suffering from AD worldwide [21]. And it was estimated that 5 million Americans aged 65 and older have $\mathrm{AD}$ in 2013. By 2050 this figure is expected to exceed 115 million worldwide while it is projected that 14 million Americans will live with AD by 2050. Perhaps in China the situation will be even worse as the population of aging people will be more [26].

\subsection{RISK FACTORS AND IMPACT ON SOCIETY}

Along with the Age and genetics of $\mathrm{AD}$, there are various risk factors which are responsible for cause of AD. AD affects physically, financially, and emotionally to the patients and their families [25].

\subsection{SUPPORTING PATHOLOGICAL TESTS ALONG WITH NEUROIMAGING TECHNIQUES}

The clinical diagnosis of Alzheimer's is based on the investigation of the medical history of patients, physical examinations, clinical lab tests and neuropsychological tests like Mini Mental State Examination (MMSE), Clinical Dementia Rate (CDR), Geriatric Depression Scale (GDS) that measure memory, attention, language skills and problem-solving abilities. Severe cognitive deficit and autopsy confirmation of histopathological changes in the brain confirms the diagnosis of AD. Along with neuropsychological tests, there are some other Neuroimaging techniques which plays very important role in diagnosis of $\mathrm{AD}$ such as Computed Tomography (CT), Magnetic Resonance Imaging (MRI), Positron Emission Tomography (PET), Single Photon Emission Computed Tomography (SPECT), Functional Magnetic Resonance Imaging (fMRI), Electroencephalogram (EEG), Magneto Encephalogram (MEG) etc.

\subsection{NEED FOR EARLY DIAGNOSIS}

Early diagnosis of $\mathrm{AD}$, particularly that of MCI is important as in early stage medical treatments are more effective and can help to improve the quality of life of the patients; it also helps researchers to deeply understand the causes of the disease to slow down the progress of $\mathrm{AD}$ and offers more chances to treatments in the early stages. However this is only possible with accurate diagnosis of $\mathrm{AD}$ in its early symptomatic stage. All patients with different $\mathrm{AD}$ stages have specific atrophic changes in the temporal lobes of the brain [21].

\section{LITERATURE SURVEY}

The modern way of Neuroimaging techniques have enabled researchers to analyze and quantify different structures and functions of the brain. These imaging techniques aid in the detection of AD. Donghai Guan et al [22] have presented detail survey on mislabeled training data detection techniques and provide comparison of different classifiers. Yazdani et al. [32] have provided detail survey of segmentation techniques for brain MRI which might prove to be helpful in AD cases. Norouzi et al. [29] in their paper have covered many segmentation methods for Medical Image Analysis covering advantages and disadvantages of each with application to MRI and CT. Automatic classification using various machine learning methods, segmentation of diseased tissues or other anatomical parts of brain, feature extraction and selection methods has significant impact for detection of the onset and progression of this disease. For this reason feature extraction and selection methods and classification methods can also be incorporated in the detection of AD. In this section (Refer Table.1) an in-depth literature study is conducted to comprehend

- MRI Neuroimaging technique, in particular and

- Pattern recognition techniques for AD Detection

The Table.1 summarizes the previous work done with respect to the use of classifier, type of training data used, number of classes considered and the results obtained in terms of parameters like Sensitivity, Specificity, and Accuracy etc. Here Accuracy is measured as total number of correctly classified images divided by the total number of images; In short, it is the fraction of correctly classified instances. Sensitivity measures the percentage of actual positive (may be AD) instances which are correctly identified. Specificity measures the percentage of negative (normal) instances which are correctly identified.

As summarized in Table.1, approaches used for classification falls into two categories:

i. Supervised learning techniques such as ANN [16], SVM $[5-7,9,11,14,16-18,21,23,31]$ and K-NN $[16,21]$ etc.

ii. Unsupervised learning techniques such as $\mathrm{K}$ - means clustering, Self-Organizing Map (SOM) [41] etc.

Class labels are considered as NC (Normal Controls/Cases), $\mathrm{MCI}$ and $\mathrm{AD}$. Following section of the literature survey describes some of the methods implemented by researchers/authors in brief: J. H. Morra et al. [9] compared four automated methods for hippocampal segmentation using different machine learning algorithms

(1) hierarchical AdaBoost, 
(2) Support Vector Machines (SVM) with manual feature selection,

(3) hierarchical SVM with automated feature selection (AdaSVM), and

(4) publicly available brain segmentation package; and shown that all methods are capable of capturing both disease related effects and correlations between cognition and structure for the well-known, widespread effects.

Y. Fan [12] proposed an ordinal ranking based classification method for distinguishing NC, MCI non-converter (MCI-NC), $\mathrm{MCI}$ converter (MCI-C), and $\mathrm{AD}$ at an individual level, taking into account the inherent ordinal severity of brain damage caused by normal aging, MCI, and $\mathrm{AD}$, rather than formulating the classification as a multi-class classification problem.

D. Zhanga et al. [14] proposed a new multimodal data fusion and classification method based on kernel combination for AD and MCI. Compared with the conventional direct feature concatenation method, this method provides a unified way to combine heterogeneous data, particularly for the case where different types of data cannot be directly concatenated.

J. Rajeesh et al. [18] made an analysis, whether the texture features of hippocampus on MRI can be used as a biomarker to identify $\mathrm{AD}$ and gave analysis that shows proper selection of texture features can discriminate the AD from Normal from hippocampus texture features.

M.Liu et al. [19] proposed a hierarchical ensemble classification algorithm to gradually combine the features and decisions into a unified model for more accurate classification. Specifically, a number of low-level classifiers are first built to transform the rich imaging and correlation-context features of brain image into more compact high-level features with supervised learning. Then, multiple high-level classifiers are generated, with each evaluating the high-level features of different brain regions. Finally, all high-level classifiers are combined to make final decision.

G. Wiselin et al. [23] compared two automated methods for hippocampal segmentation using different machine learning algorithms: 1) support vector machines (SVM) with manual feature selection, 2) hierarchical SVM with automated feature selection (Ada-SVM). Also evaluated how segmentation accuracy depended on the size of the training set, providing practical information for future users of this technique.

E. Westman et al. [24] demonstrated that combining raw cortical thickness measures with sub-cortical volumes normalized by intracranial volume gives the best prediction accuracy for separating AD subjects from cognitively normal subjects. Andrea Rueda et al. [30] presented a new fully automatic image analysis method that reveals discriminative brain patterns associated to the presence of neurodegenerative diseases, mining systematic differences and therefore grading objectively any neurological disorder. He found that main changes are located in horizontal and diagonal directions, indirect evidence that changes occur very likely in oriented areas but not precisely located in a particular region. With an adequate and exhaustive evaluation in larger data sets, containing sufficient examples of the different AD stages, saliency based pattern recognition can be also used as a second diagnostic opinion in the current clinical practice.
Q. Zhou et al. [31] proposed method to combine MRI data with a neuropsychological test, Mini Mental State Examination (MMSE), as input to a multi-dimensional space for the classification of Alzheimer's disease (AD) and it's prodromal stages ,Mild Cognitive Impairment (MCI) including amnestic MCI (aMCI) and non-amnestic MCI (naMCI).

W. Yang presented [38] his work in five steps: preprocessing of MR images, segmentation of gray matter of the brain, decomposition using independent component analysis, extraction of voxel of interest, and classification by a support vector machine classifier for classification of MRI scans of Alzheimer patients and healthy subjects.

C. Tanchi [40] presented new automatic method to segment the whole brain in Magnetic Resonance (MR) image series and calculated its volume for detecting Alzheimer's disease (AD). He also proposed the three-class classification problem on the data set using the Bayes classifier and four-fold cross validation. S.T. Yang [41] used PCA for feature extraction and compared the performance of the 2 classifiers i.e. SVM and SOM classifiers, for the classification of $\mathrm{AD}, \mathrm{MCI}$ and NC subjects from MRI data sets collected from Chang Gung Memorial Hospital, Lin-Kou Taiwan.

Al-Naami [42] proposed a fusion method to distinguish between the normal and (AD) MRIs. 27 MRIs collected from Jordanian Hospitals are analyzed based on the use of Low pass morphological filters to get the extracted statistical outputs through intensity histogram to be employed by the descriptive box plot. Also, the artificial neural network (ANN) is applied to test the performance of this approach.

Y. Zhang [43] proposed a novel classification system to distinguish among elderly subjects with AD, MCI, and NC. First, all these three dimensional (3D) MRI images were pre-processed with atlas-registered normalization. Then, gray matter images were extracted and the 3D images were under-sampled. Afterwards, principle component analysis (PCA) was applied for feature extraction. On the basis of the extracted features, author constructed a kernel support vector machine decision tree (kSVMDT).

\section{NEUROIMAGING TECHNIQUES USED DIAGNOSIS OF AD}

Neuroimaging examinations are an essential part of the diagnostic investigation of dementia. These techniques allows the study of the brain in living subjects and thus are not only highly important research tools, but are effective in order to improve the accuracy of clinical diagnosis and identify brain lesions contributing to the cognitive decline in dementia cases. A variety of Neuroimaging techniques, including CT scan, Structural MRI, fMRI, SPECT, PET, EEG, MEG [27] etc. have made significant advances during the last years in an attempt to diagnose and monitor the progression of the AD. The Table. 2 provides detail comparison of different Neuroimaging techniques.

\section{PATTERN RECOGNITION TECHNIQUES}

Pattern recognition involves the design of new algorithms for recognition of complex patterns, with its application in 
handwriting recognition, speech recognition, fingerprint recognition, biometrics, stock market analysis, medical diagnosis and many more. The key to pattern recognition for medical image analysis problems is the extraction of features from a variety of Neuroimaging techniques (Table.2) and then its classification. A large number of techniques exist in literature for extraction of features. Similarly an enormous number of classification methods (Table.3) can be found in literature that can perform with high accuracy.

\subsection{FEATURE EXTRACTION AND SELECTION}

Features are used as inputs to classifiers which assign them to the class that they represent. There different types of features like shape based, color based, texture based [37], wavelet based [42], region based, histogram based, GLCM based [37], etc. are extracted from the brain image for the diagnosis of AD. As summarized in Table.1, in semi / fully automated AD classification systems, statistical features [1, 4, 18, 21, 31], Haralick's textural features [18, 37], Hippocampal volume based features (indicators of atrophy) [4, 8, 10, 13, 14, 24] are amongst the widely used features.

Feature extraction enable to reduce the original data by measuring certain properties of images which have relevant data, or features, that distinguish one pattern from another pattern.
The feature extraction stage is designed to obtain a compact, non-redundant and meaningful representation of observations. These features are used by the classifier to classify the data. It is assumed that a classifier that uses smaller and relevant features will provide better accuracy and require less memory and improves the computational speed of the classifier [16]. After feature extraction, only some of the features from the dataset are selected and used in the training process of the learning algorithm. In this process the aim is to find the optimal subset that increases the efficiency of the learning algorithm.

Feature selection (FS) algorithms [45] are based on the approach to reduce dimension by finding the "best" least subset of the original features, without transforming the data to a new set of dimensions.

Features can be selected using filter method, wrapper method [44], Sequential forward selection and backward elimination method, correlation based method, mutual information based method and wavelet based techniques.

\subsection{CLASSIFICATION TECHNIQUES}

The various methods for classification of Neuroimages for detection AD include K-NN [21,16], SVM [5, 6, 7, 9, 11, 16, 18, 21, 23, 31], Adaboost [9], Naïve Bayes [21], PCA, ICA, LDA, ANN, Decision tree, fuzzy technique, Logistic Regression, etc. which gives the best results for basic feature extraction used for the diagnosis of AD as listed in Table.3.

Table.1. Review of MRI Neuroimaging and Pattern Recognition Techniques.

\begin{tabular}{|c|c|c|c|c|c|c|c|c|}
\hline Classifier Used & $\begin{array}{l}\text { No of } \\
\text { Images }\end{array}$ & $\begin{array}{c}\text { Source of } \\
\text { Image }\end{array}$ & Features & \multicolumn{5}{|c|}{ Results } \\
\hline \multirow[b]{2}{*}{$\begin{array}{c}\text { Stepwise } \\
\text { Discriminant } \\
\text { Analysis [1] }\end{array}$} & $\mathrm{NC}=40$ & \multirow[b]{2}{*}{$\begin{array}{l}\text { Onset } \\
\text { Dating }\end{array}$} & \multirow{2}{*}{\begin{tabular}{|c|} 
Total-260 \\
Sequential \\
Forward Selection \\
Variance, \\
Entropy \\
\end{tabular}} & & Acc & Sen & Spe & \\
\hline & $\mathrm{AD}=24$ & & & & $91 \%$ & $79 \%$ & $100 \%$ & \\
\hline \multirow{5}{*}{$\begin{array}{c}\text { Discrimination } \\
\text { Function and } \\
\text { Multivariate } \\
\text { Analysis of Variance } \\
\text { [2] }\end{array}$} & $\mathrm{NC}=32$ & $\begin{array}{l}\text { NINCDS- } \\
\text { ADRDA }\end{array}$ & $\begin{array}{l}\text { Hippocampal } \\
\text { Volume, }\end{array}$ & Volumes & Acc $\%$ & Sen $\%$ & Spe $\%$ & \\
\hline & $\mathrm{AD}=30$ & & $\begin{array}{l}\text { Entrohinal } \\
\text { Thickness }\end{array}$ & $\mathrm{HC}$ & 86 & 80 & 91 & \\
\hline & & & & EC & 87 & 80 & 94 & \\
\hline & & & & HC \& gender & 90 & 87 & 94 & \\
\hline & & & & EC \& gender & 92 & 90 & 94 & \\
\hline \multirow{4}{*}{$\begin{array}{c}\text { Analysis of Variance } \\
{[3]}\end{array}$} & \multirow{4}{*}{$\begin{array}{c}\mathrm{NC}=42 \\
\mathrm{MCI}=123\end{array}$} & \multirow{4}{*}{ Print Media } & & & & & \multicolumn{2}{|c|}{ Acc } \\
\hline & & & & \multicolumn{3}{|c|}{ Controls From Converters } & \multicolumn{2}{|c|}{$89 \%$} \\
\hline & & & & \multicolumn{3}{|c|}{ Controls \& questionable } & \multicolumn{2}{|c|}{$74 \%$} \\
\hline & & & & \multicolumn{3}{|c|}{ Questionable \& Converters } & \multicolumn{2}{|c|}{$80 \%$} \\
\hline \multirow{4}{*}{$\begin{array}{l}\text { Logistic Regression } \\
\text { Analysis [4] }\end{array}$} & \multirow{4}{*}{$\begin{array}{c}\mathrm{NC}=63, \\
\mathrm{MCI}=139\end{array}$} & \multirow{4}{*}{\begin{tabular}{|c|} 
NINCDS- \\
ADRDA \\
Memory \\
Disorders \\
Centre at \\
New York \\
\end{tabular}} & \multirow{4}{*}{\begin{tabular}{|c|} 
Hippo-campus and \\
EC \\
Volumes, Mean, \\
Std \\
Deviation, \\
Percentage \\
\end{tabular}} & \multicolumn{5}{|c|}{ HC \& EC Volumes } \\
\hline & & & & \multicolumn{3}{|c|}{$\mathrm{NC}$} & \multicolumn{2}{|c|}{ Largest } \\
\hline & & & & \multicolumn{3}{|c|}{ naMCI } & \multicolumn{2}{|c|}{ Intermediate } \\
\hline & & & & \multicolumn{3}{|c|}{$\mathrm{AD}$} & \multicolumn{2}{|c|}{ Smallest } \\
\hline \multirow{3}{*}{ SVM [5] } & \multirow{3}{*}{$\begin{array}{l}\mathrm{NC}=22, \\
\mathrm{AD}=16\end{array}$} & \multirow{3}{*}{$\begin{array}{l}\text { Onset } \\
\text { Dating }\end{array}$} & \multirow{3}{*}{ Mean } & \multicolumn{5}{|c|}{ Correct Classification } \\
\hline & & & & $\mathrm{AD}$ & \multicolumn{4}{|c|}{ Mean $=94.50 \%$} \\
\hline & & & & $\mathrm{NC}$ & \multicolumn{2}{|c|}{ Spe $=96.6 \%$} & \multicolumn{2}{|c|}{ Sen $=91.5 \%$} \\
\hline
\end{tabular}




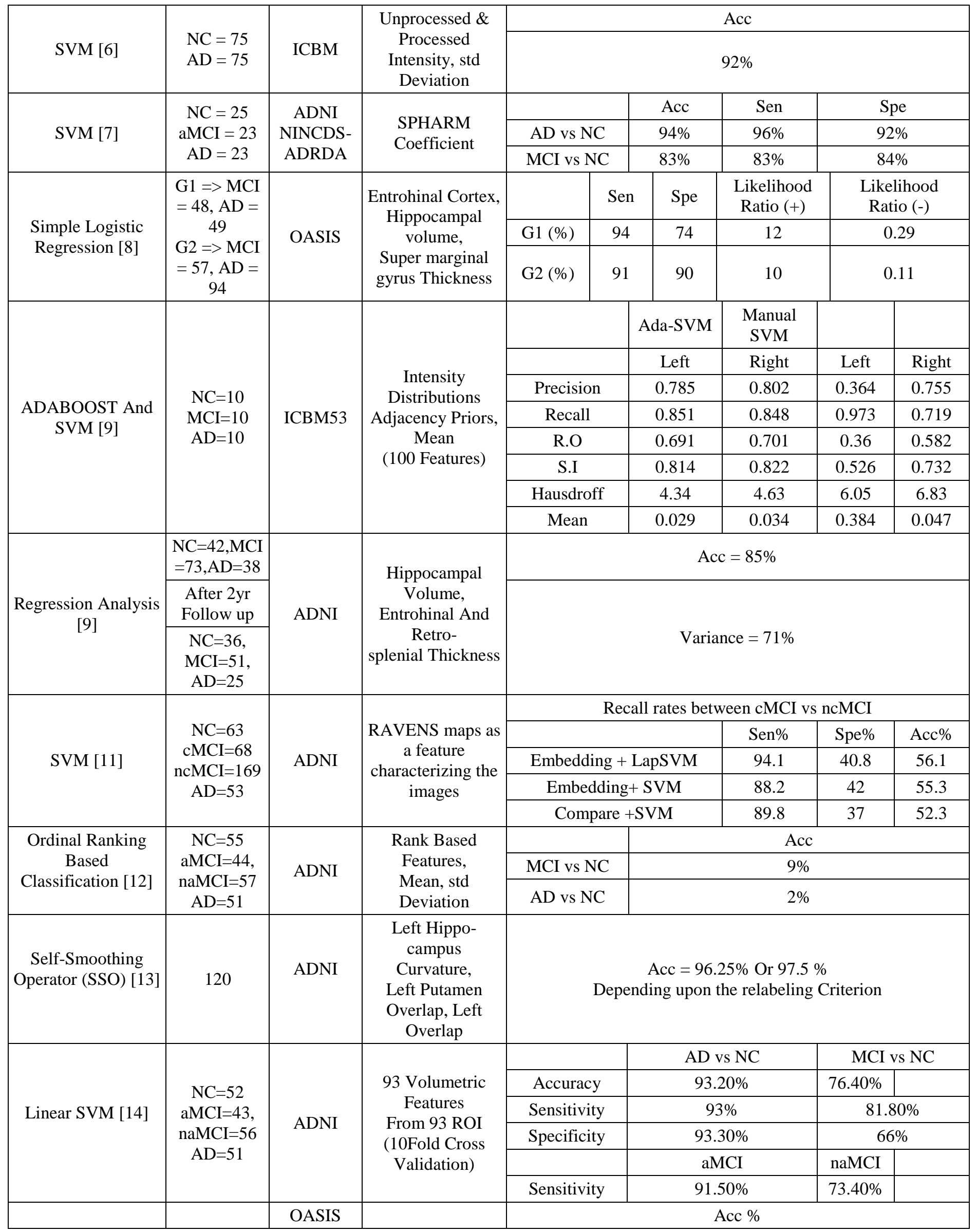




\begin{tabular}{|c|c|c|c|c|c|c|c|c|}
\hline \multirow{4}{*}{$\begin{array}{c}\text { SVM, KNN, ANN } \\
{[16]}\end{array}$} & \multirow{4}{*}{$\begin{array}{c}\mathrm{NC}=83 \\
\mathrm{MCI}=62 \\
\mathrm{AD}=25\end{array}$} & & \multirow{4}{*}{$\begin{array}{c}\text { ICA and PCA are } \\
\text { used }\end{array}$} & & NC \&MCI & NC\&AD & \multicolumn{2}{|c|}{ MC\&AD } \\
\hline & & & & KNN & 58.04 & 64.71 & \multicolumn{2}{|c|}{68.06} \\
\hline & & & & ANN & 53.57 & 40.2 & \multicolumn{2}{|c|}{41.67} \\
\hline & & & & PSVM & 47.19 & 58.67 & \multicolumn{2}{|c|}{60.65} \\
\hline $\begin{array}{l}\text { Regularized Logistic } \\
\text { Regression, } \\
\text { Linear SVM, Linear } \\
\text { Classifier [17] }\end{array}$ & $\begin{array}{c}\mathrm{NC}=205 \\
\mathrm{MCI}=351 \\
\mathrm{AD}=171\end{array}$ & ADNI & $\begin{array}{l}\text { PCA is used. } \\
\text { Machine } \\
\text { Learning } \\
\text { Algorithm, } \\
\text { SNR, } \\
\text { Sample Size }\end{array}$ & \multicolumn{5}{|c|}{ Classification Accuracy Increased as Sample Size Increased } \\
\hline \multirow{5}{*}{ SVM [18] } & \multirow{5}{*}{$\begin{array}{l}\mathrm{NC}=146 \\
\mathrm{AD}=133\end{array}$} & \multirow{5}{*}{ ADNI } & \multirow{5}{*}{\begin{tabular}{|c|} 
Textural Features- \\
Entropy, \\
Variance, \\
Skewness, \\
Symmetry, \\
Mean (69 Features)
\end{tabular}} & & Case1 & Case2 & Case3 & Case4 \\
\hline & & & & Precision & 90.90 & 88.90 & 89.10 & 95.30 \\
\hline & & & & Sen & 88.90 & 88.90 & 91.90 & 91.10 \\
\hline & & & & Spe & 91.80 & 89.80 & 89.80 & 95.90 \\
\hline & & & & Acc & 90.40 & 89.40 & 90.40 & 93.60 \\
\hline $\begin{array}{l}\text { SVM, Ada-AVM } \\
\text { [23] }\end{array}$ & \begin{tabular}{|c|} 
Training \\
AD,MCI,NC \\
$=10$ \\
Testing \\
AD,NC $=20$
\end{tabular} & ICBM & \begin{tabular}{|c|} 
Intensities, \\
Gradients, \\
Curvatures, Tissue \\
classify. \\
Local filters, \\
Spectral \\
Decomposition, \\
etc.
\end{tabular} & \multicolumn{5}{|c|}{$\begin{array}{l}\text { Adaboost and Ada-SVM gives Superior accuracy than the } \\
\text { SVM Classifier Methods }\end{array}$} \\
\hline \multirow{5}{*}{$\begin{array}{c}\text { KNN, SVM, Naïve } \\
\text { Bayes [21] }\end{array}$} & \multirow{5}{*}{$\begin{array}{l}\mathrm{NC}=255 \\
\mathrm{AD}=72\end{array}$} & \multirow{5}{*}{ OASIS } & \multirow{5}{*}{\begin{tabular}{|c|} 
Color Mean, \\
Image Symmetry, \\
Center Black Area, \\
Total Brain Area, \\
Image \\
Mean, \\
Image Variance, \\
Skewness kurtosis, \\
Gradient \\
\end{tabular}} & \multicolumn{5}{|c|}{ Method } \\
\hline & & & & K-NN & & $\begin{array}{l}\text { Normal } \\
\text { Results \% }\end{array}$ & Strict $\mathrm{F}$ & sults $\%$ \\
\hline & & & & SVM & & 85.3 & & \\
\hline & & & & Naive Bay & & 83.5 & & \\
\hline & & & & & & 80.7 & & \\
\hline & & & Regional Volume, & & & Acc & & \\
\hline & $\mathrm{NC}$ & & Cortical Thickness, & & & $\mathrm{s} \mathrm{NC}$ & $\mathrm{MCI}$ & $\mathrm{AD}$ \\
\hline $\begin{array}{l}\text { Multivariate } \\
\text { Analysis [24] }\end{array}$ & $\begin{array}{c}\mathrm{MCI}=287 \\
\mathrm{AD}=187\end{array}$ & ADNI & $\begin{array}{c}\text { Volume, } \\
\text { Surface Area, } \\
\text { Mean } \\
\text { Curvature }\end{array}$ & $\begin{array}{l}\text { Classifier } \\
\text { Methods: } \\
\text { Single }\end{array}$ & & $50 \%$ & & \\
\hline Single classifier, & & & & $\begin{array}{c}\text { Ensemble low } \\
\text { level }\end{array}$ & Acc $\%$ & Sen $\%$ & Spe $\%$ & AUC \\
\hline ensemble low level & $\begin{array}{l}\mathrm{NC}=229 \\
\mathrm{AD}-189\end{array}$ & ADNI & Correlation contex & Multiple & 86.43 & 83.89 & 88.64 & 0.928 \\
\hline $\begin{array}{c}\text { classit1er, Multilevel } \\
\text { Classifier [19] }\end{array}$ & & & & Parameter & 89.7 & 86.89 & 92.11 & 0.939 \\
\hline & & & & Acc & 92.04 & 90.92 & 92.98 & 0.951 \\
\hline & G1 $=>$ & & & Sen & G1 & G2 & G3 & G4 \\
\hline & $\mathrm{NC}=66$ & & & Spe & 86.05 & 80.16 & 76.47 & 70.2 \\
\hline & $\begin{array}{c}\mathrm{MCl}=20 \\
\mathrm{G} 2=>\mathrm{NC}=98\end{array}$ & & Intensity, & BAC & 85 & 75 & 87.14 & 70 \\
\hline Based Pattern &, $\mathrm{MCI}=28$ & $\begin{array}{l}\text { OASIS- } \\
\text { MIRIAD }\end{array}$ & Orientation, & F-Measure & 86.36 & 81.63 & 69.7 & 73.47 \\
\hline Recognition [30] & $\mathrm{G} 3=>\mathrm{NC}=66$ & & $\begin{array}{l}\text { Contrast } \\
\text { (18Features) }\end{array}$ & EER & 85.68 & 78.32 & 76.28 & 70.23 \\
\hline & $\begin{array}{l}, \mathrm{MCl}=70 \\
\mathrm{G} 4=>\mathrm{NC}=\end{array}$ & & & & 73.91 & 62.29 & 78.71 & 69.65 \\
\hline & $98, \mathrm{MCI}=100$ & & & & 0.86 & 0.79 & 0.79 & 0.69 \\
\hline SVM [31] & $\mathrm{NC}=59$ & Private & & & Acc & Sen & & \\
\hline
\end{tabular}




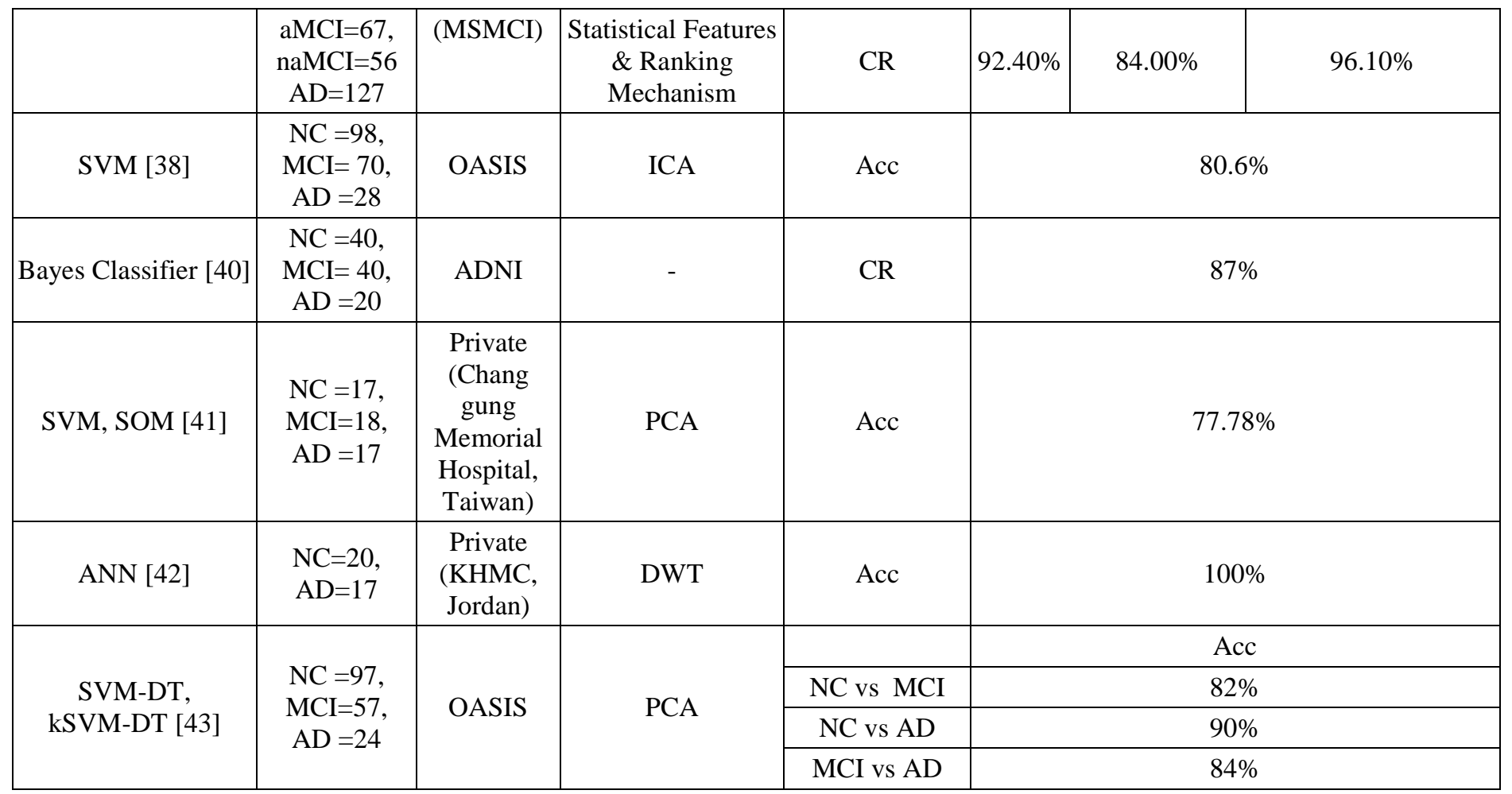

Acc: Accuracy, Sen: Sensitivity, Spe: Specificity, CR: Classification Rate, HC: Hippocampus, EC: Entrohinal Cortex, NC: Normal Control, MCI: Mild Cognitive Impairment, AD: Alzheimer's Disease, SVM: Support Vector Machine, KNN: K-Nearest Neighbor, ANN: Artificial Neural Network, OASIS: Open Access Series for Imaging Studies [34], ADNI: Alzheimer's Disease Neuroimaging Initiative [35], NINCDS-ADRDA: National Institute of Neurological and Communicative Disorders and Stroke-Alzheimer's Disease and Related Disorders Association, ICBM: International Consortium for Brain Mapping, MIRIAD: Minimal Interval Resonance Imaging in Alzheimer's Disease

Table.2. Comparison of Neuroimaging Techniques used in the diagnosis of AD

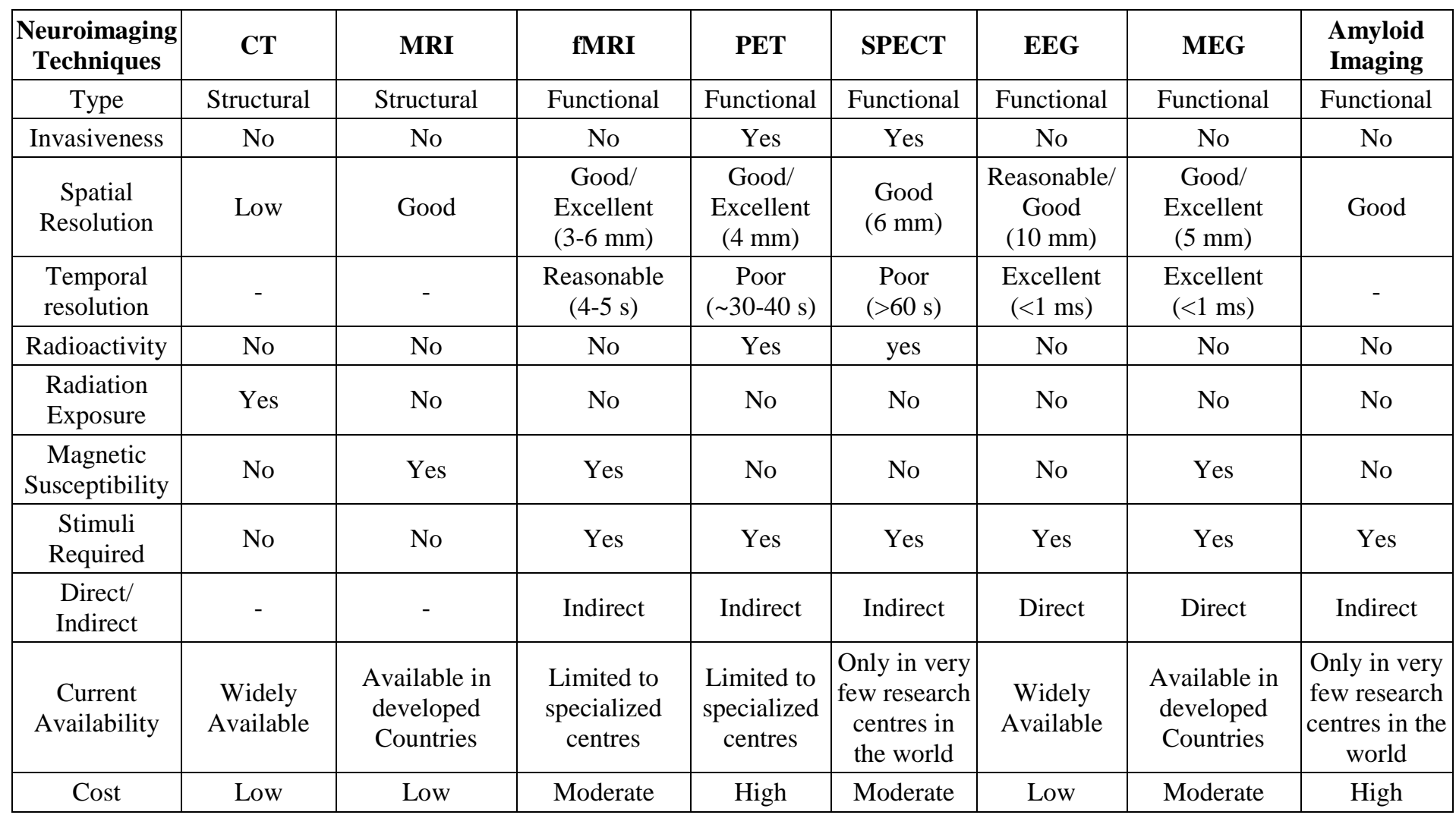




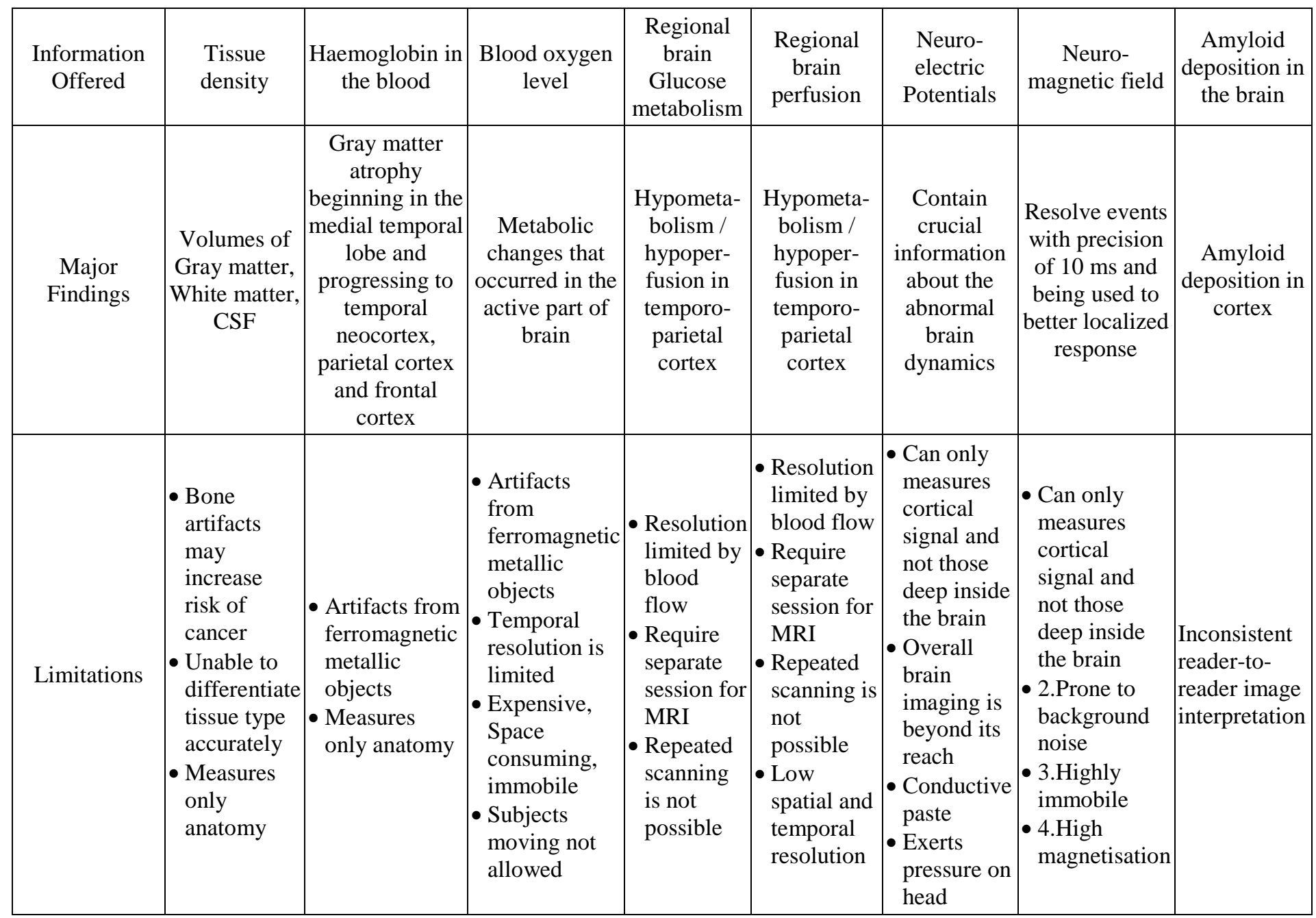

Table.3. Some Relatively Good Classification Techniques used in the Detection of AD. K-NN: K-Nearest Neighbor, SVM: Support Vector Machine, ICA: Independent Component Analysis, PCA: Principal Component Analysis, LDA: Linear Discriminant Analysis, ANN: Artificial Neural Network

\begin{tabular}{|c|c|c|c|}
\hline $\begin{array}{l}\text { Classification } \\
\text { Techniques }\end{array}$ & Key Points & Advantages & Disadvantages \\
\hline $\mathrm{K}-\mathrm{NN}$ & $\begin{array}{l}\text { Labelled data, distance measure and } \\
\text { no of nearest neighbour }\end{array}$ & $\begin{array}{l}\text { Simple, robust to noisy training } \\
\text { data, less design complexity, no } \\
\text { training time }\end{array}$ & $\begin{array}{l}\text { Large memory and recognition time, } \\
\text { low accuracy in multidimensional } \\
\text { data with irrelevant features, no } \\
\text { thumb rule for determination of } \mathrm{k} \text {. }\end{array}$ \\
\hline SVM & $\begin{array}{l}\text { Separates both linear and non-linear } \\
\text { data, Kernel trick used for non-linear } \\
\text { data, hyper plane, support vectors }\end{array}$ & $\begin{array}{c}\text { Minimizes no of misclassifications, } \\
\text { independent of feature } \\
\text { dimensionality, accurate results }\end{array}$ & $\begin{array}{c}\text { Limitations in size and speed during } \\
\text { testing and training, solution depend } \\
\text { on selection of kernel, depend on } \\
\text { size of data }\end{array}$ \\
\hline Ad boost & $\begin{array}{l}\text { Weight of samples, weak classifier, } \\
\text { error rate, no of iterations }\end{array}$ & $\begin{array}{l}\text { Fast and easy to program, no } \\
\text { parameters to tune, no prior } \\
\text { knowledge, flexible with weak } \\
\text { classifiers, mathematically } \\
\text { insensitive to over-training }\end{array}$ & $\begin{array}{c}\text { Boosting depend on input data and } \\
\text { weak classifier, Susceptible to noise, } \\
\text { failed with insufficient data }\end{array}$ \\
\hline PCA & $\begin{array}{c}\text { Dimensionality reduction, Eigen } \\
\text { vectors, Co-variance of set of images, } \\
\text { lossless transformation }\end{array}$ & $\begin{array}{c}\text { Correlation between components of } \\
\text { the data vector be clearly viewed } \\
\text { and it capture the second order } \\
\text { correlation value }\end{array}$ & $\begin{array}{c}\text { Identifies the linear combinations of } \\
\text { variable and ignore the high order } \\
\text { correlation value }\end{array}$ \\
\hline ICA & $\begin{array}{l}\text { Probabilistic and multivariate } \\
\text { method, independent and non- }\end{array}$ & $\begin{array}{l}\text { It captures the high order statistics } \\
\text { of the data }\end{array}$ & $\begin{array}{l}\text { If the data sources are independent } \\
\text { then it works well }\end{array}$ \\
\hline
\end{tabular}




\begin{tabular}{|c|c|c|c|}
\hline & $\begin{array}{l}\text { Gaussian component search, linear } \\
\text { transform of random vector }\end{array}$ & & \\
\hline LDA & $\begin{array}{c}\text { Maximizes the ratio of between-class } \\
\text { variance to within-class variance, } \\
\text { Gaussian distribution and equal } \\
\text { covariance matrix }\end{array}$ & $\begin{array}{l}\text { Linear mapping, the number of } \\
\text { classes of the data limits the } \\
\text { dimensionality of the subspace }\end{array}$ & $\begin{array}{l}\text { Data handling is difficult when the } \\
\text { individual classes are far from } \\
\text { Gaussian and has small sample size }\end{array}$ \\
\hline ANN & $\begin{array}{l}\text { Network structure, momentum rate, } \\
\text { learning rate, converging criteria, } \\
\text { Efficient with few input variables }\end{array}$ & $\begin{array}{l}\text { High parallel ability and fast } \\
\text { computation, noise toleration }\end{array}$ & $\begin{array}{l}\text { Difficult to understand structure, } \\
\text { optimal network structure } \\
\text { determined by experimentation }\end{array}$ \\
\hline
\end{tabular}

\section{DISCUSSION}

We have presented number of Neuroimaging and pattern recognition techniques and a comprehensive literature survey of AD identification using high dimensional pattern classification methods in Table.1. With the advancements in Neuroimaging technology and the development of new imaging techniques, search for precise, cheap and non-invasive techniques have been significantly evaluated and compared with respect to different aspects in Table.2. Neuroimaging techniques like PET with high spatial resolution are preferred. PET imaging technique is also capable to capture the metabolic activity deep inside the brain as opposed to MEG and EEG. Similarly we need to know the anatomical structures of the brain to identify the regions of interest. For this purpose MRI is considered to be the best. The datasets including MRI, PET, SPECT images, used for evaluation, can be obtained from the OASIS [35], ADNI [36], ICBM, NINCDS-ADRDA and MIRIAD. In the section of pattern recognition, we have presented automatic classification techniques using different feature extraction and selection methods and various classifiers which are used in the diagnosis of AD (Table.3).

\section{CONCLUSION}

There is no single pattern recognition technique which gives consistent results for all types of neuroimages. The performance of pattern recognition techniques depends on the type and size of the experimental dataset. So, one can use hybrid or integrated pattern recognition technique such as fusion of different classifiers for achieving better performance. The feature extraction and selection and type of features to be selected have wide scope of research in AD detection. Achieving $100 \%$ classification for $\mathrm{AD}$ is definitely a challenge. Based on $\mathrm{AD}$ progression (from Normal Controls (NC) - MCI - AD) different classification models such as NC versus $\mathrm{MCI}$, MCI versus AD and NC versus AD can be considered. Optimal features based on anatomical changes (possible atrophy) in case of AD can be one of the key research interests in medical image analysis community.

\section{ACKNOWLEDGMENT}

Authors are thankful to Dr. Rohit Sangolkar, Nizam's institute of Medical Sciences, Hyderabad and Dr. Geetanjali Kadam, Dinanath Mangeshkar Hospital Pune for their valuable guidelines related to Alzheimer's disease required during this review work.

\section{REFERENCES}

[1] P.A. Freeborough and N.C. Fox, "MR Image Texture Analysis Applied to the Diagnosis and Tracking of Alzheimer's Disease", IEEE Transactions on Medical Imaging, Vol. 17, No. 3, pp. 475-478, 1998.

[2] Kirsi Juottonen, Mikko P. Laakso, Kaarina Partanen and Hilkka Soininen, "Comparative MR Analysis of the Entorhinal Cortex and Hippocampus in Diagnosing Alzheimer Disease", American Journal of Neuroradiology, Vol. 20, No. 1, pp. 139-144, 1999.

[3] M.S. Albert, M.B. Moss, R. Tanzi and K. Jones, "Preclinical Prediction of AD using Neuropsychological Tests", Journal of the International Neuropsychological Society, Vol. 7, No. 5, pp. 631-639, 2001.

[4] D.P. Devanand, X. Liu, A. Khandji, S.D. Santi, S. Segal, H. Rusinek, G.H. Pelton, L.S. Honig, R. Mayeux, Y. Stern, M.H. Tabert and M. J. Leon, "Hippocampal and Entrohinal Atrophy In Mild Cognitive Impairment Prediction of Alzheimer Disease", Neurology, Vol. 68, No. 11, pp. 828836, 2007.

[5] B. Magnin, L. Mesrob, S. Kinkingnehun, M. P.-Issac, O. Colliot, M. Sarazin, B. Dubois, S. Lehericy, and H. Benali, "Support Vector Machine-based Classification of Alzheimer's Disease from Whole-Brain Anatomical MRI", Neuroradiology, Vol. 51, No. 2, pp. 73-83, 2008.

[6] Simon Duchesne, Anna Caroli, C. Geroldi, Christian Barillot, Giovanni B. Frisoni and D. Louis Collins, "MRIBased Automated Computer Classification of Probable AD Versus Normal Controls", IEEE Transactions on Medical Imaging, Vol. 27, No. 4, pp. 509-520, 2008.

[7] E. Gerardin, "Multidimensional Classification of Hippocampal Shape Features Discriminates Alzheimer's Disease and Mild Cognitive Impairment from Normal Aging”, Neuroimage, Vol. 47, No. 4, pp. 1476-1486, 2009.

[8] R.S. Desikan et al., "Automated MRI Measures Identify Individuals with Mild Cognitive Impairment and Alzheimer's Disease", American Journal of Neuroradiology, Vol. 132, No. 8, pp. 2048-2057, 2009.

[9] J.H. Morra, Z. Tu, L.G. Apostolova, A.E. Green, A.W. Toga and P.M. Thompson, "Comparison of Ada Boost and Support Vector Machines for Detecting Alzheimer's Disease through Automated Hippocampal Segmentation”, IEEE Transactions on Medical Imaging, Vol. 29, No. 1, pp. 30-43, 2010.

[10] K.B. Walhovd et.al., "Combining MR Imaging, PositronEmission Tomography, and CSF Biomarkers in the Diagnosis and Prognosis of Alzheimer Disease", American 
Journal of Neuroradiology, Vol. 31, No. 2, pp. 347-354, 2010.

[11] D.H. Ye, K.M. Pohl and C. Davatzikos, "Semi-Supervised Pattern Classification: Application to Structural MRI of Alzheimer's Disease", Proceedings of IEEE International Workshop on Pattern Recognition in Neuroimaging, pp. 14, 2011.

[12] Y. Fan, “Ordinal Ranking for Detecting Mild Cognitive Impairment and Alzheimer's Disease based on Multimodal Neuroimages and CSF Biomarkers", Proceedings of International Workshop on Multimodal Brain Image Analysis, pp. 44-51, 2011.

[13] J.E. Iglesias, J. Jiang, C. Liu and Z. Tu, "Classification of Alzheimer's Disease using a Self-Smoothing Operator", Proceedings of International Conference on Medical Image Computing and Computer-Assisted Intervention, pp. 58-65, 2011.

[14] D. Zhanga, Y. Wanga, L. Zhoua, H. Yuana and D. Shen, "Multimodal Classification of Alzheimer's Disease and Mild Cognitive Impairment", Neuroimage, Vol. 55, No. 3, pp. 856-867, 2011.

[15] R. Umer, "Machine Learning Approaches for the Computer Aided Diagnosis and Prediction of Alzheimer's Disease Based on Clinical Data", PhD Dissertation, Department of Computer Science, University of Georgia, 2011.

[16] A.B. Tufail, A. Abidi, A.M. Siddiqui and M.S. Younis, "Automatic Classification of Initial Categories of Alzheimer's Disease from Structural MRI Phase Images: A Comparison of PSVM, KNN and ANN Methods", World Academy of Science, Engineering and Technology, Vol. 6, No. 12, pp. 1570-1574, 2012.

[17] R. Casanova, F.C. Hsu and M.A. Espeland, "Classification of Structural MRI Images in Alzheimer's Disease from the Perspective of III-Posed Problems", Worldwide Alzheimer's Disease Neuroimaging Initiative, Vol. 7, No. 10, pp. 1-6, 2012.

[18] Jayapathy Rajeesh, Rama Swamy Moni and Thankappan Gopalakrishnan, "Discrimination of Alzheimer's Disease using Hippocampus Texture Features from MRI", Asian Biomedicine, Vol. 6, No. 1, pp. 87-94, 2012.

[19] M. Liu, D. Zhang, P.T. Yap and D. Shen, "Hierarchical Ensemble of Multi-level Classifiers for Diagnosis of Alzheimer's disease", Proceedings of International Workshop on Machine Learning in Medical Imaging, pp. 27 35, 2012.

[20] I.V. Maksimovich, "Certain New Aspects of Etiology and Pathogenesis of Alzheimer's Disease," Advances in Alzheimer's Disease, Vol. 1, No.3, pp. 68-76, 2012.

[21] K.S. Marcolini and S. Gillespie, "Comparing Classification Methods of MRI Brain Scans for Dementia and Alzheimer's Disease", Master Thesis, University of Miami, 2012.

[22] D. Guan, W. Yuan, "A Survey on Mislabled Training Data Detection Techniques for Pattern Classification", IETE Technical Review, Vol. 30, No. 6, pp. 524-530, 2013.

[23] G.W. Jijl and M. Rangini, "Detection of Alzheimer's Disease through Automated Hippocampal Segmentation", Proceedings of International Multi-Conference on Automation, Computing, Communication, Control and Compressed Sensing, pp. 144-149, 2013.
[24] E. Westman, C. Aguilar, J. Muehlboeck and A. Simmons, "Regional Magnetic Resonance Imaging Measures for Multivariate Analysis in Alzheimer's Disease and Mild Cognitive Impairment", Brain Topography, Vol. 26, No. 1, pp. 9-23, 2013.

[25] N. Hu, J. Tai Yu, L. Tan, Y. Wang, L. Sun and L. Tan, "Nutrition and the Risk of Alzheimer's Disease", BioMed Research International, Vol. 2013, pp. 1-12, 2013.

[26] X. Yan, "Advances in Alzheimer's Disease (AAD): Standing Firm at its First Anniversary", Advances in Alzheimer's Disease, Vol. 2, No. 2, pp. 49-50, 2013.

[27] S. Farhan, M. A. Fahiem, F. Tahir, and H. Tauseef, "A Comparative Study of Neuroimaging and Pattern Recognition Techniques for Estimation of Alzheimer's Disease", Life Science Journal, Vol. 10, No. 7, pp. 10301039, 2013.

[28] R.L. Simoes, “Towards Earlier Detection of Alzheimer's Disease using Magnetic Resonance Images", PhD Dissertation, University of Twente, 2013.

[29] A. Norouzi, M.S.M. Rahim, A. Altameem, T. Saba, Abdolvahab E. Rad, Amjad Rehman and M. Uddin, "Medical Image Segmentation Methods, Algorithms, and Application", IETE Technical Review, Vol. 31, No. 3, pp. 199-213, 2014.

[30] A. Rueda, F.A. Gonzalez and E. Romero, "Extracting Salient Brain Patterns for Imaging-Based Classification of Neurodegenerative Diseases", IEEE Transactions on Medical Imaging, Vol. 33, No. 6, pp. 1262-1274, 2014.

[31] Q. Zhou, M. Goryawala, M. Cabrerizo, J. Wang, W. Barker, D.A. Loewenstein, R. Duara and M. Adjouadi, "An Optimal Decisional Space for the Classification of Alzheimer's Disease and Mild Cognitive Impairment", IEEE Transactions on Biomedical Engineering, Vol. 61, No. 8, pp. 2245-2253, 2014.

[32] S. Yazdani, R. Yusuf, A. Karimian, M. Pasha and A. Hematian, "Image Segmentation Methods and Applications in MRI Brain Images", IETE Technical Review, Vol. 32, No. 6, pp. 413-427, 2015.

[33] J. Dauwels, F.B. Vialatte and A. Cichocki, "On the Early Diagnosis of Alzheimer's Disease from EEG Signals: A Mini-Review", Advances in Cognitive Neurodynamics, Vol. 2, pp. 709-716, 2010.

[34] Alzheimer's Society, Available at: http://www. alzheimers.org.uk.

[35] OASIS Brain Database, Available at: http://www.oasisbrain.org, Accessed on 2012.

[36] ADNI Database. Available at: http://adni.loni.usc.edu/

[37] Robert M. Haralick, K. Shanmugam and Its Hak Dinstein, "Textural Features for Image Classification", IEEE Transactions on Systems, Man, and Cybernetics, Vol. 3, No. 6, pp. 610-621, 1973.

[38] Wenlu Yang; Halei Xia, Bin Xia, Lok Ming Lui and Xudong Huang, "ICA-based Feature Extraction and Automatic Classification of AD-Related MRI Data", Proceedings of $6^{\text {th }}$ International Conference on Natural Computation, pp. 1261-1265, 2010.

[39] S.E. Fashtakeh, "Early Detection of Alzheimer's Disease using Structural MRI: A Research Idea", Life Science Journal, Vol. 9, No. 3, pp. 1072-1079, 2012. 
[40] Chaturaphat Tanchi, Nipon Theera-Umpon and Sansanee Auephanwiriyakul, "Fully Automatic Brain Segmentation for Alzheimer's", Proceedings of $6^{\text {th }}$ International Conference on Soft Computing and Intelligent Systems, pp. 1393-1396, 2012.

[41] S.T. Yang, "Discrimination between Alzheimer's disease and Mild Cognitive Impairment using SOM and PSOSVM", Computational and Mathematical Methods in Medicine, Vol. 2013, pp. 1-10, 2013.

[42] B. Al-Naami, N. Gharaibeh and A. Kheshman, "Automated Detection of Alzheimer's Disease using Region Growing Technique and Artificial neural Network", World Academy of Science Engineering and Technology, Vol. 7, No. 5, pp. 204-208, 2013.
[43] Y. Zhang, S. Wang and Z. Dong, "Classification of Alzheimer Disease Based on Structural Magnetic Resonance Imaging by Kernel Support Vector Machine Decision Tree", Progress In Electromagnetic Research, Vol. 144, pp. 171184, 2014.

[44] R. Kohavi and G.H. John, "Wrappers for Feature Subset Selection”, Artificial Intelligence, Vol. 97, No. 1-2, pp. 273324, 1997.

[45] A. Jain and D. Zongker, "Feature Selection: Evaluation, Application, and Small Sample Performance", IEEE Transactions on Pattern Analysis and Machine Intelligence, Vol. 19, No. 2, pp. 153-158, 1997. 